\title{
Linking Synaptic Computation for Image Enhancement
}

\author{
Kun Zhan ${ }^{\mathrm{a}}$, Jinhui Shi ${ }^{\mathrm{a}}$, Jicai Teng ${ }^{\mathrm{a}}$, Qiaoqiao $\mathrm{Li}^{\mathrm{b}}$, Mingying $\mathrm{Wang}^{\mathrm{a}}$, \\ Fuxiang $\mathrm{Lu}^{\mathrm{a}}$ \\ ${ }^{a}$ School of Information Science and Engineering, Lanzhou University, Lanzhou, Gansu, \\ 730000, China \\ ${ }^{b}$ Faculty of Systems Science and Technology, Akita Prefectural University, Honjo, Akita, \\ 015-0055, Japan
}

\begin{abstract}
Linking synaptic computation network is proposed. The linking synapse is introduced into the neural network inspired by the gamma band oscillations in visual cortical neurons, and the neural network is applied to image representation. The linking synaptic mechanism of the network allows integrating temporal and spatial information. An image is input to the network and the enhanced result is obtained by the final linking synaptic state. The visual performance of the results boosts the details while preserving the information in the input image. The effectiveness of the method has been borne out by five quantitative metrics as well as qualitative comparisons with other methods.
\end{abstract}

Keywords: Image enhancement, neural networks, PCNN, LSCN, synaptic computation

\section{Introduction}

The gamma band oscillations in the primary visual cortex is discovered by Eckhorn et al. [1] and Gray et al. [2] independently, which is considered to be a significant progress in visual neuroscience [3-5]. After the discovery of the gamma band oscillations, Eckhorn et al. explain the synchronous activity of primate cortical neurons by a linking field model [6], and it is simplified to pulse-coupled neural networks (PCNN) [7-9]. As PCNN is developed directly from the studies of visual cortical properties, PCNN has become a general and powerful tool for image processing [9-11]. Based on the studies of the aforementioned models, we propose the linking synaptic computation 
network (LSCN) in this paper. As Eckhorn et al. introduce the crucial modulatory linking synapse which is inspired by the gamma band oscillations [6], LSCN emphasizes the linking synapse which has neurophysiological support. Using individual spikes allows integrating temporal and spatial information in synaptic computation as well as real neurons do [12]. We use the temporal and spatial integration effect of the linking synapse for image enhancement.

The aim of image enhancement is to improve the visual appearance evaluated by human visual perception, and enhancement is useful when image contrast is imperceptible or barely perceptible [13-15]. The most well-known image enhancement method is the classic histogram equalization because of its simplicity of implementation. The histogram based methods always produce inadequate detail preservation or an excessively enhanced image [16-21]. The transform domain methods achieve enhancement by boosting the coefficients of high-frequency subbands or magnifying the measured contrast [22-24]. However, the parameters of these methods are not suitable to every general image and these methods also magnify the noisy intensity. The linear scale-space theory supports structure-preserving while suppressing noise [25], and there are many improved structure-preserving smoothing techniques are proposed recently, such as bilateral filter [26], weighted least squares filter [27], $L_{0}$-smoothing filter [28], guided filter [29], etc. If these structure-preserving filters are applied to image enhancement, they usually suffer from "halo" artifacts around the major structures [29-31]. The human visual system (HVS) is powerful in enhancing a scene with precise representation of contrast, and there are many image enhancement methods are inspired from HVS [32-34]. Spiking cortical model (SCM), a variant of $\mathrm{PCNN}$, is applied to image enhancement and its processing mechanism is consistent with HVS $[35,36]$. Weber-Fechner's law and the Mach band effect are simulated well in SCM method. However, the results of SCM enhancement algorithm suffer from contrast degradation in bright regions and some pixels with the lowest intensity change to white.

We propose an image enhancement method based on mechanism of the linking synaptic computation. The goal of the image enhancement method is to improve visibility while preserving the information within the input image. Image details are enhanced by using the neural mechanisms related to the linking synapse. We conduct experiments to compare the proposed method with methods based on histogram equalization [37], SCM [35, 36], generalized equalization model [21], and gradient distribution specification [38]. The experiment results illustrate that the LSCN-based method is ef- 
fective in image enhancement as well as in preserving the information of the input image.

The contributions of this paper are summarized as follows:

1. A neural network LSCN is designed.

2. A general image enhancement framework is proposed.

3. A network iterative stopping condition is proposed.

4. We find that the final linking synaptic state is related with the stimulus image.

\section{Linking Synaptic Computation Network}

\subsection{Leaky Integrator}

The dynamic potential $v(t)$ of a neural oscillator is described via a leaky integrator,

$$
\frac{\mathrm{d} v(t)}{\mathrm{d} t}=-a v(t)+s
$$

where $t$ is time, $s$ is the input and $a$ is the leak rate.

The potential (1) can be discretized as,

$$
\frac{V(n)-V(n-1)}{n-(n-1)}=-a V(n-1)+s
$$

where $V(n)$ is the discretized potential, and $n$ is the discrete time.

We can rewrite (2) as,

$$
V(n)=b V(n-1)+s
$$

where $b$ is the attenuation constant of the leaky integrator.

\section{2. $L S C N$}

The linking synapse, the membrane potential and the threshold are instantiated as leaky integrators.

The postsynaptic potential feeds back to modulate the linking synapse. The linking synapse is represented by a leaky integrator [6-9],

$$
L_{i j}(n)=l L_{i j}(n-1)+\sum_{p q} W_{i j p q} Y_{p q}(n-1)
$$


where each neuron is denoted with indices $(i, j)$, one of its neighboring neurons is denoted with $(p, q), l$ is the linking constant, $W_{i j p q}$ is the weight applied to a linking synapse and $Y_{p q}(n-1)$ is the postsynaptic action potential.

Cortical networks have both feedback and feedforward components and the feedforward component integrates the stimulus-driven neuronal input $[6,12,39]$. The two components are combined together to produce the membrane potential. In this paper, the membrane potential is represented by a leaky integrator,

$$
U_{i j}(n)=f U_{i j}(n-1)+S_{i j}\left(1+\beta L_{i j}(n)\right)
$$

where $f$ is the membrane potential attenuation constant, $S_{i j}$ carries the stimulus information and $\beta$ is the linking strength.

The threshold is an evolution from the neuron analog in [40]. The absolute and relative refractory period are simulated well by the threshold [40]. The threshold of a neuron is represented by a leaky integrator $[6,40]$.

$$
\Theta_{i j}(n)=g \Theta_{i j}(n-1)+h Y_{i j}(n-1)
$$

where $g$ is the threshold attenuation constant, $h$ is a magnitude adjustment, and $Y_{i j}(n-1)$ is the postsynaptic action potential.

At the beginning of the network iteration, the threshold decays from the initial value $\Theta_{i j}(0)$ before the first spike occurs,

$$
\Theta_{i j}(n)=g^{n} \Theta_{i j}(0) .
$$

Threshold can be replaced with a linear decay function [9],

$$
\Theta_{i j}(n)=\Theta_{i j}(n-1)-\delta+h Y_{i j}(n-1)
$$

where $\delta$ is a positive small constant.

Similarly, the threshold decays from the initial value $\Theta_{i j}(0)$ before the first spike occurs,

$$
\Theta_{i j}(n)=\Theta_{i j}(0)-n \delta
$$

When the membrane potential of a neuron exceeds its threshold in the 
network iteration, the neuron produces a spike,

$$
Y_{i j}(n)= \begin{cases}1, & \text { if } U_{i j}(n)>\Theta_{i j}(n) \\ 0, & \text { otherwise }\end{cases}
$$

The linking synaptic computation network (LSCN) is described by (4), (5), (6), and (10). The schematic of LSCN is shown in Fig. 1.

LSCN retains two significant properties in the linking field network [6]. The first is that the linking synapse is represented by the leaky integrator $[1,6]$ and the second is the dynamic threshold [6, 40]. The two properties of LSCN are also the significant differences from the conventional integrateand-fire model. LSCN has two differences from PCNN, the first is that the membrane potential is represented by the leaky integrator, and the second is that the feeding input is simplified to the stimulus only. The modifications are under the consideration that the membrane potential of the most biological neural networks is represented by the leaky integrator and the main contribution of feeding input is the stimulus [6]. The main difference from SCM is that LSCN represents the linking synapse as a leaky integrator.

As the linking strength $\beta$ is usually set to a small value [9-11], we assume that it is set to 0 and obtain Fig. 2 based on (5) and (9).

The firing condition is that the membrane potential is larger than the decaying threshold. As can be seen from Fig.2, the spike timing is when the membrane potential is almost equal to the threshold,

$$
\Theta_{i j}(n)=U_{i j}(n)
$$

Once a neuron fires the first spike, in the next iteration of the network the threshold is changed to,

$$
\Theta_{i j}(n+1)=g U_{i j}(n)+h,
$$

then the threshold delays exponentially according to its attenuation constant $g$. The second and the following spikes produce when the threshold is almost equal to the membrane potential again, so the firing cycle $C_{i j}$ of a neuron is given by,

$$
C_{i j}=\log _{g}\left(\frac{U_{i j}}{g U_{i j}+h}\right) .
$$




\subsection{Multiple Pass}

The proposed multiple pass working form of LSCN is a network stopping condition. The stopping condition is how to set the iterative times of the network. The iterative times is usually set manually [9-11]. Different from the conventional PCNN, the iterative process is automatic stop rather than manually set the iterative times in the proposed multiple pass form.

The multiple pass stopping condition is that the network stops when all neurons in the network produce their spikes and it is given by Algorithm 1 .

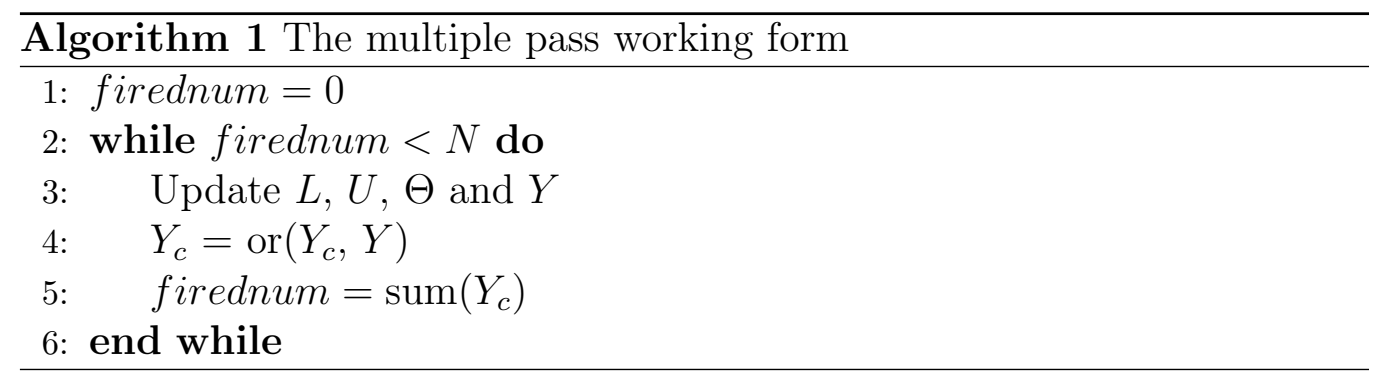

In Algorithm 1, $N$ is the total number of neurons in a network, firednum is counting the number of the fired neurons in each iteration, $Y_{c}$ with initial values of 0 has the same size of $Y$, and the function 'or' performs an elementby-element 'or' operator between matrices $Y_{c}$ and $Y$.

\section{Image Enhancement}

\subsection{Noise Reduction}

Gonzalez et al. [37] introduce an adaptive local noise reduction filter by using mean $\mu_{w}$ and variance $\sigma_{w}^{2}$ in a sliding window $w$,

$$
\bar{S}_{i j}=S_{i j}+\frac{\sigma_{\eta}^{2}}{\sigma_{w}^{2}}\left(\mu_{w}-S_{i j}\right), \forall(i, j) \in w
$$

where $S_{i j}$ is an input image at location $(i, j), \bar{S}_{i j}$ is the smoothed output image, $\sigma_{\eta}^{2}$ denotes the estimated variance of noise within the input image.

(14) is equivalent to the adaptive local Wiener filter [41-43] and the filter is given by,

$$
\bar{S}_{i j}=\mu_{w}+\frac{\sigma_{w}^{2}-\sigma_{\eta}^{2}}{\sigma_{w}^{2}}\left(S_{i j}-\mu_{w}\right), \forall(i, j) \in w
$$


Let $k$ denote $\frac{\sigma_{w}^{2}-\sigma_{\eta}^{2}}{\sigma_{w}^{2}}[41]$, then,

$$
\bar{S}_{i j}=\mu_{w}+k\left(S_{i j}-\mu_{w}\right), \forall(i, j) \in w .
$$

In image enhancement, the intensity scale of noisy pixels is less than edges and corners but larger than the intensity scale of flat patches [37], so we want to minimize an optimization problem in the sliding window $w$,

$$
\min _{k} \sum_{(i, j) \in w}\left(\left(\bar{S}_{i j}-S_{i j}\right)^{2}+\lambda k^{2}\right)
$$

where $\lambda$ a regularization parameter penalizing large $k$.

(17) is the linear ridge regression model and its solution is given by

$$
k=\frac{\sigma_{w}^{2}}{\sigma_{w}^{2}+\lambda}
$$

If we tone the regularization parameter $\lambda$ in a range of the intensity scale of noise, the noisy pixels in flat patches can be smoothed by the filter [29]. i.e., if $S_{i j}$ changes a lot within the window $w$, we have $\sigma_{w}^{2}>>\lambda$, so $k=1$, then $\bar{S}_{i j}=S_{i j}$. If $S_{i j}$ is almost constant in a window $w$ located at a flat patch, we have $\sigma_{w}^{2}<<\lambda$, so $k=0$, then $\bar{S}_{i j}=\mu_{w}$. In this way, the main structure (e.g. edges and corners) can be preserved and noise in flat regions can be smoothed. $\lambda$ is set to 0.2 in this paper.

\subsection{Image Enhancement Algorithm}

Each neuron corresponds to one pixel in an input image and the intensity of the pixel is regarded as an external stimulus.

At first, an input image $I$ with the intensity in the range $[0,255]$ is normalized to

$$
S=\frac{I-\min (I)}{\max (I)-\min (I)}+\varepsilon
$$

where $\min (I)$ returns the minimum value of $I, \max (I)$ returns the maximum value of $I$, and $\varepsilon$ is set to a small positive value $\frac{1}{255}$ in this paper so that the threshold decays to a lower value than $\varepsilon$ and even neurons with lowest stimuli fire finally.

The stimulus $S$ is the input of LSCN, and the output of the enhanced image is the final linking synaptic state $L$. 
As the stimulus $S$ is the main input of the membrane potential $U$, we assume that the membrane potential $U$ is equal to the stimulus $S$ and we rewrite (13) as,

$$
C_{i j}=\log _{g}\left(\frac{S_{i j}}{g S_{i j}+h}\right) .
$$

From (20), we can obtain a conclusion that a neuron with higher stimulus has a shorter firing cycle. Note that, by (4), its final state of $L_{i j}$ is a higher value for a single neuron with a shorter firing cycle. The firing spike $Y_{i j}$ frequently integrates into the state of $L_{i j}$ and the final state of $L_{i j}$ has an approximate proportionality relationship with $S_{i j}$.

Besides the firing cycle, the final linking state is also related to the first spiking time because the integration effect of $L_{i j}$ is activated after the first spike $Y_{i j}$ fires generally.

The parameter $f$ is generally set to a unified constant and all the elements in the membrane potential matrix are initialized to 0 [9-11]. So the first firing time of neurons are only decided by the initial value of the threshold.

As the starting point of the linking synaptic integration is decided by the initial value of the threshold, we can add edge-enhanced effect by,

$$
\Theta_{i j}(0)=1+S_{i j} * \mathcal{L}
$$

where $\mathcal{L}$ is the $3 \times 3$ Laplacian operator and $*$ is the convolution operator.

An edge in an image is similar to the first line in Fig.3, and the initial threshold effect (21) can be seen as the second line in Fig.3. As shown in Fig.2, when the threshold is set as the second line in Fig.3, neurons at the lower side of the edge fire later than normal and neurons at the higher side of the edge fire earlier than normal. The final states of $L$ become smaller in the lower side of the edge but larger in the higher side of the edge, which is a good simulation of the Mach band effect.

Considering (20), the higher intensity pixels usually fires earlier than the lower intensity. If $\Theta_{i j}(0)$ is initialized as (21), the second term may returns a value less than -1 , these pixels fire earlier than others and their intensity may belong to a dark region. In output image, these pixels may change to a high intensity and they may look like noisy pixels. To solve this problem, we modify (21) and make sure that all pixels fires in descending order,

$$
\Theta_{i j}(0)=1+d_{i j}-\min \left(d_{i j}\right)
$$


where $d_{i j}=S_{i j} * \mathcal{L}$ denotes the details within an image.

The details can also be obtained by,

$$
d_{i j}=\bar{S}_{i j}-S_{i j}
$$

where $\bar{S}_{i j}$ is a smoothed image of $S_{i j}$.

(23) has same effect to $S_{i j} * \mathcal{L}$ [37], which is easy to verify

$$
\begin{aligned}
\bar{S}_{i j}-S_{i j} & =\frac{1}{9} S_{i j} *\left[\begin{array}{ccc}
1 & 1 & 1 \\
1 & 1 & 1 \\
1 & 1 & 1
\end{array}\right]-S_{i j} *\left[\begin{array}{ccc}
0 & 0 & 0 \\
0 & 1 & 0 \\
0 & 0 & 0
\end{array}\right] \\
& =\frac{1}{9} S_{i j} * \mathcal{L}
\end{aligned}
$$

where $\mathcal{L}$ is given by,

$$
\mathcal{L}=\left[\begin{array}{ccc}
1 & 1 & 1 \\
1 & -8 & 1 \\
1 & 1 & 1
\end{array}\right]
$$

Because HVS has lower contrast sensitivity for dark region than bright region, the contrast in the dark region should be enhanced more than bright region. Thus, we modify the details term to,

$$
d_{i j}=\kappa\left(\bar{S}_{i j}-S_{i j}\right)\left(1-S_{i j}\right)
$$

where the constant $\kappa$ is used to control the magnitude of the details and it is set to 9 in this paper.

Note that $\bar{S}_{i j}$ is obtained by (16) and (18) in our algorithm, so the details can be enhanced and noise reduction is also considered [44, 45].

The network is implemented with Algorithm 1. The output image is obtained by,

$$
\mathcal{J}=\left\lfloor 255 \frac{L-\min (L)}{\max (L)-\min (L)}+0.5\right\rfloor .
$$

In order to eliminate the influence of the sparse pixels with over high or over lower intensity, we optimize $\mathcal{J}$ to new intensities in $J$ such that $2 \%$ of pixels are saturated at low and high intensities of $\mathcal{J}$. Let $M$ denote the saturated high intensity of $\mathcal{J}$ and $m$ the saturated low intensity. The 
intensities are adjusted by,

$$
J= \begin{cases}0, & \mathcal{J}<m \\ \left\lfloor 255 \frac{\mathcal{J}-m}{M-m}+0.5\right\rfloor, & \mathcal{J} \in[m, M] \\ 255, & \mathcal{J}>M .\end{cases}
$$

Finding the best solution of the pair $[m, M]$ is an optimization problem of the form,

$$
\begin{array}{ll}
\min & M-m \\
\text { s.t. } & \sum_{\mathcal{J} \in[m, M]} P(\mathcal{J}) \geqslant 98 \%
\end{array}
$$

where $P(\mathcal{J})$ is the probability of occurrence of the intensity grayscale $\mathcal{J}$.

The optimization is to find the intensity values $m$ and $M$, which makes the intensity interval $[m, M]$ occupy a given percentage $98 \%$ in the original intensity occurrence probability. The variable $m$ varies from 0 to a certain intensity $\mathcal{J}_{m}$ whose cumulative distribution probability satisfies,

$$
\sum_{\mathcal{J} \in[0, m]} P(\mathcal{J})=2 \%
$$

In this way, we narrow down the searching range of $m$. In the same way, the searching range of $M$ is narrowed down too. After the searching ranges are achieved, the difference of each latent pair $[m, M]$ is obtained and the desired pair with the minimum difference can be found.

\subsection{Computational Complexity Analysis}

Some steps of the image enhancement algorithm involve taking 2-D convolution. Given an $r \times c$ image with intensity in the range $[0, R-1]$ has $\log _{2} R$ bits for a grayscale image and filter window $w \times w$ (i.e. the synaptic weight matrix), LSCN is efficient with computational complexity $O\left(w^{2} r c\right)$ in one iteration. In the multi-pass working form, the network usually iterates $R$ times in order to output the same dynamic range with the input image. So the LSCN algorithm has complexity $O\left(R w^{2} r c\right)$. The step of initializing threshold needs calculate the local mean and local variance in a $3 \times 3$ window, so it has complexity $O\left(2 \times 3^{2} r c\right)$. The last gray stretch step needs compute 
the Histogram, so it has complexity $O(r c)$. The overall complexity of the image enhancement algorithm is $O\left(R w^{2} r c+19 r c\right)$.

\section{Experimental Results}

\subsection{Experimental Setup}

The proposed method is compared with the histogram equalization method (HEM) [37], the SCM based method [35, 36], the generalized equalization model based method (GEM) [21], and the gradient distribution specification method (GDS) [38]. The parameters of HEM, SCM, GEM, and GDS are set to the values given by the respective authors.

The parameters of LSCN is determined by its working form. We use the multi-pass working form, and the output image is obtained by the final state of linking term. In order to obtain similar dynamic range, we set $\delta$ to $\frac{1}{255}$. If $\delta=\frac{1}{255}$, LSCN iterates approximately 256 times and the output image is expected to have the dynamic range of $[0,255]$. Because the final state of $L_{i j}$ is a higher value for a single neuron with a shorter firing cycle and the linking term records the firing rate of neurons, so we set $l=1$ and the center element of synaptic weight matrix is set to 1 as given by,

$$
W=\left[\begin{array}{ccc}
0.04 & 0.04 & 0.04 \\
0.04 & 1 & 0.04 \\
0.04 & 0.04 & 0.04
\end{array}\right]
$$

In order to record the firing rate preciously, we set $h$ to $0.001\left(<\frac{1}{255}\right)$. Because LSCN neurons fire synchronously in a local-connected region using recruitment based on stimulus similarity and spatial proximity, an image can be smoothed by LSCN $[35,46]$. The degree of image smooth is controlled by the linking term of LSCN [9] so the other synaptic weights except center one are set to 0.04. Since the stable value of the membrane potential of LSCN is equal to $\frac{S_{i j}}{1-f}$ approximately [35], we set $f$ to a small value 0.01 so that the stable value is less than the initialized threshold. The linking term is a weak modulation term for the membrane potential and the main contribution of the membrane potential is the stimulus, so we set the linking strength $\beta$ to a small value $0.01[9-11]$.

If an image with a size of $r \times c$ is input, $N$ is equal to $r \times c$ in Algorithm 1. We use the linear decay function for the threshold (8). 


\subsection{Quantitative Metrics}

In order to evaluate the enhancement performance of different methods objectively, five quantitative metrics, i.e., local contrast-based metric $\left(Q_{c}\right.$ $[47])$, spatial frequency-based metrics $\left(Q_{\mathrm{sf}}[48]\right)$, gradient-based metrics $\left(Q_{g}\right.$ $[49])$, visibility level-based metrics $(\bar{r}[50])$ and JND-based metrics $\left(Q_{\text {jnd }}[51]\right)$ are adopted. The default parameters given by the respective authors are adopted for these quality metrics.

1. Average Local Contrast $\left(Q_{c}\right)$ : The contrast metrics $Q_{c}$ of output image is obtained by computing the average value of the local contrast metrics. Variance can be used to evaluate the image contrast [52]. To quantitatively evaluate the image enhancement algorithms in terms of contrast enhancing, we use the average of a local contrast metric [47]. The local contrast metric is defined in a sliding $3 \times 3$ window $w$,

$$
C_{i j}=\frac{\sigma_{w}^{2}}{\mu_{w}}
$$

where the local mean of intensity $\mu_{w}$ is computed for the pixels within the sliding $3 \times 3$ window centered on the pixel $(i, j)$, and $\sigma_{w}^{2}$ is the local variance of intensity.

2. Spatial Frequency $\left(Q_{\mathrm{sf}}\right)$ : The spatial frequency measures the overall activity level in an image [48]. The spatial frequency is defined as follows,

$$
Q_{\text {sf }}=\sqrt{\text { Row_F } F^{2}+\text { Column_F }}
$$

where Row_F and Column_F are the row and column frequencies, and they are given by,

$$
\begin{gathered}
\text { Row_F }=\sqrt{\frac{1}{N} \sum_{i=1}^{r} \sum_{j=2}^{c}\left[J_{i, j}-J_{i, j-1}\right]^{2}}, \\
\text { Column_F }=\sqrt{\frac{1}{N} \sum_{j=1}^{r} \sum_{i=2}^{c}\left[J_{i, j}-J_{i-1, j}\right]^{2} .}
\end{gathered}
$$

3. Average Gradient $\left(Q_{g}\right)$ : The average gradient can evaluate the edge information of enhanced images [49], they are also used to evaluate the 
enhancement algorithms. The average gradient is given by,

$$
Q_{g}=\frac{1}{N} \sum_{j=1}^{r} \sum_{i=1}^{c} G_{i j}
$$

where $G_{i j}$ represents the magnitude of the gradient at the pixel location $(i, j)$ in an image.

4. Geometric Mean of the Ratios of Visibility Level $(\bar{r})$ : This metric returns the ratio of visibility level of the considered object in the output enhanced image and in the input image [50]. The value of $\bar{r}$ expresses the quality of the contrast enhancement and the definition of $\bar{r}$ is out of the range of our paper. The details of the $\bar{r}$ implementation have been given in [50] and the code of $\bar{r}$ are available at the authors' website ${ }^{1}$.

5. JND-based Valid Perception Ratio $\left(Q_{\text {jnd }}\right)$ : A just noticeable distortion (JND) threshold $T$ is derived based on the local average brightness and local spatial frequency $[51,53,54]$. The effect of enhancement is evaluated through a comparison of local brightness $\Delta B$ and the JND threshold $T$,

$$
Q_{\text {jnd }}=\frac{\sum \mathrm{H}(D(\Delta B-T))}{\sum D}
$$

where $D$ is a decision map of image structure and is calculated by using Canny detector; $\mathrm{H}(\cdot)$ is the Heaviside function.

\subsection{Experimental Results}

Five input images are selected to evaluate the enhancement techniques. Two gray images man and tire are from MATLAB image toolbox, two color images boys and deer are from Berkeley Segmentation Data Set [55], and the last color image flower is from [27]. In the experiments, we enhance the gray-scale images directly, or enhance value channel of the HSV model of color images. We compare LSCN with other baseline enhancement methods. Input images, with their corresponding processed results, are shown in Figs. $4,5,6,7$, and 8, respectively.

The results of HEQ and GEM methods are similar because they are both histogram based techniques. The results of them have a loss of details generating the blur effect, as shown in Figs. 5(b), 5(d), 6(b), 6(d), 7(b), and

\footnotetext{
${ }^{1}$ http://perso.lcpc.fr/tarel.jean-philippe/visibility/EvaluationDescriptor.zip
} 
7(d). Also, the sky in Figs. 4(b) and 4(d) has obvious white region. HEQ and GEM methods enhance contrast globally, so they are not suitable for enhancing every image.

As shown in Figs. 5(c) and 8(c), SCM based method renders some dark regions change to white. Some pixels with the intensity of 0 change to white after the inverse operation of the time matrix because their thresholds cannot decay to 0 and they cannot fire [35]. The results of SCM boost too much the intensity so that the images are over enhanced, as shown in Figs. 4(c), $6(\mathrm{c})$ and $7(\mathrm{c})$. The existing problems within the SCM method are solved well in LSCN method. In SCM method, the darkest pixels are not fired which change to white after the inverse operation for the SCM time matrix. This problem does not exist in LSCN method because LSCN even processes the darkest pixels in multiple pass working form. SCM processes the dark pixels too much and makes the whole image becomes too bright.

Comparing Figs. 4(e), 5(e), 6(e), and 8(e) with their corresponding input images, we can find that GDS obtains weak enhancement effect. Fig. 8(e) is more bright than Fig. 8(a), but the background within it changes to black blocks, which means that the original information is lost.

LSCN method obtains good visual performance than others, and the other results produce more or less distortion. Comparing with other methods, LSCN method boosts much more details in the results, and the edges in the results of LSCN method are more clear, which can be seen in Figs. 4(f), $5(\mathrm{f}), 6(\mathrm{f}), 7(\mathrm{f})$ and $8(\mathrm{f})$. However, there are some potential problems in LSCN algorithm, e.g., there are some intrinsic noisy pixels in each image, and LSCN recognizes them as details of the image and enhances these pixels. We extract edges of the images to show the effect easily. Given an image, the edges are obtained by $G=|J * \mathcal{L}|$, and $\mathcal{L}$ denotes the Laplacian operator (25). Results show in Fig. 9. More edges are visible after the enhancement processing of LSCN, which indicates that subtle details are enhanced but few noisy pixels are also easy to be found.

The quantitative metrics are summarized in Table 1. The grayscale intensity or the value channel of the HSV model is used to evaluate the performance. LSCN method almost obtains all the largest metric, so we can say that the objective quantitative evaluation is consistent with the subjective visual effect of the enhanced images.

We compare LSCN with other image enhancement method with respect to runtime. We run each method 1000 times and report the average runtime. The result is in Table 2. Unsurprisingly, HEQ always runs fastest for its 
Table 1: Quantitative Metrics of Different Algorithms

\begin{tabular}{l|l|ccccc}
\hline Metric & Image & HEQ & SCM & GEM & GDS & LSCN \\
\hline \multirow{4}{*}{$Q_{c}$} & man & 1.2971 & 1.2352 & 1.2154 & $\mathbf{3 . 7 3 6 2}$ & 2.9669 \\
& tire & 3.4615 & 5.8633 & 3.1042 & 3.1348 & $\mathbf{8 . 6 1 3 3}$ \\
& boys & 2.9290 & 0.7421 & 2.5690 & 1.1416 & $\mathbf{7 . 8 8 6 6}$ \\
& deer & 1.4557 & 0.6501 & 1.1047 & 0.9460 & $\mathbf{3 . 0 2 4 1}$ \\
& flower & 0.5713 & 0.1219 & 0.4597 & $\mathbf{1 . 5 9 8 9}$ & 1.4272 \\
\hline \multirow{5}{*}{$Q_{\text {sf }}$} & man & 16.5423 & 17.8442 & 15.0825 & $\mathbf{2 4 . 4 5 8 3}$ & 24.0989 \\
& tire & 27.7017 & $\mathbf{4 8 . 2 9 4 7}$ & 22.6101 & 19.9757 & 43.4376 \\
& boys & 25.4990 & 21.2891 & 24.1521 & 17.1837 & $\mathbf{4 5 . 8 3 3 9}$ \\
& deer & 21.5237 & 19.9948 & 18.2391 & 16.9766 & $\mathbf{2 8 . 0 7 0 3}$ \\
& flower & 10.0084 & 8.0510 & 9.1475 & 14.9398 & $\mathbf{1 6 . 1 0 7 4}$ \\
\hline \multirow{5}{*}{$Q_{g}$} & man & 7.5419 & 7.1079 & 7.0817 & 9.2005 & $\mathbf{1 0 . 1 3 4 5}$ \\
& tire & 14.7928 & 18.9433 & 12.7750 & 10.3877 & $\mathbf{2 0 . 5 6 2 8}$ \\
& boys & 12.7531 & 8.7186 & 12.1218 & 8.6171 & $\mathbf{2 1 . 0 9 5 1}$ \\
& deer & 10.3468 & 6.7889 & 9.1258 & 8.4652 & $\mathbf{1 1 . 9 5 0 4}$ \\
& flower & 4.8252 & 2.7674 & 4.5245 & 5.0411 & $\mathbf{7 . 1 9 9 2}$ \\
\hline \multirow{5}{*}{$Q_{\text {jnd }}$} & man & 2.1221 & $\mathbf{2 . 8 3 8 6}$ & 1.6421 & 2.1463 & 2.5121 \\
& tire & 3.0279 & $\mathbf{3 . 2 4 6 3}$ & 1.8747 & 1.0000 & 2.5040 \\
& boys & 1.2716 & 0.9934 & 1.1719 & 0.8181 & $\mathbf{2 . 1 4 8 7}$ \\
& deer & 1.6101 & 1.7336 & 1.3455 & 1.2478 & $\mathbf{1 . 7 7 4 1}$ \\
& flower & 1.5253 & 1.0698 & 1.3031 & 2.1340 & $\mathbf{2 . 1 8 4 4}$ \\
\hline \multirow{6}{*}{} & mays & 0.2306 & 0.3041 & 0.2204 & 0.3244 & $\mathbf{0 . 3 5 9 6}$ \\
& deer & 0.2432 & 0.2166 & 0.1998 & 0.1843 & $\mathbf{0 . 2 5 0 9}$ \\
& flower & 0.1649 & $\mathbf{0 . 2 6 8 2}$ & 0.1500 & 0.1530 & 0.2333 \\
\hline & & & & & &
\end{tabular}


simple implementation. By contrast, the two neural networks based methods (SCM and LSCN) run relatively slow. This is because they need iteration for obtaining a high gray level resolution.

Table 2: Runtime of Different Algorithms (s)

\begin{tabular}{lccccc}
\hline Image & HEQ & SCM & GEM & GDS & LSCN \\
\hline man & 0.0073 & 0.5858 & 0.1101 & 0.0550 & 1.4941 \\
tire & 0.0013 & 0.0853 & 0.0223 & 0.0232 & 0.2637 \\
boys & 0.0043 & 0.3360 & 0.0710 & 0.0474 & 1.3391 \\
deer & 0.0041 & 0.3016 & 0.0648 & 0.0433 & 0.9869 \\
flower & 0.0110 & 1.0282 & 0.1826 & 0.0834 & 2.7309 \\
\hline
\end{tabular}

More experiments are conducted on Ehinger et al. 's dataset (Ehinger) [56], Extended Complex Scene Saliency Dataset (ECSSD) [57], Zurich Buildings Database ${ }^{2}(\mathrm{ZuBuD})$, and 8 Scene Categories Dataset (8-Scenes) [58]. LSCN, with other compared methods, has been applied into the 912 images in Ehinger, the 1000 images in ECSSD, the 1005 images in $\mathrm{ZuBuD}$, and the 2688 images in 8-Scenes, totally 5605 images to demonstrate its effectiveness.

We calculate the average performance of different methods in each database and the best performance rate of LSCN method for each database. The results are listed in Table 3 and Table 4. As shown in Table 3, LSCN method outperforms the other methods in terms of $Q_{c}, Q_{\text {sf }}, Q_{g}, \bar{r}$, and $Q_{\text {jnd }}$. Table 4 indicates that LSCN method well enhances most images in the four mentioned databases.

\section{Conclusions}

A general framework for image enhancement is presented. The presented framework employs the study on the linking synaptic computation. The mechanism of the linking synaptic computation is inspired by the gamma band oscillations via local coupled synaptic modulation in visual cortex [6]. A multiple pass network stopping condition is proposed. By using the proposed image enhancement method, the contrast of the processed image is improved without introducing visual artifacts. Results illustrate that the

\footnotetext{
${ }^{2}$ http://www.vision.ee.ethz.ch/datasets
} 
Table 3: Average Performance in Each Database

\begin{tabular}{l|l|ccccc}
\hline Metrics & Dataset & HEQ & SCM & GEM & GDS & LSCN \\
\hline \multirow{4}{*}{$Q_{c}$} & Ehinger & 3.9159 & 1.0005 & 3.0977 & 1.6363 & $\mathbf{8 . 6 2 8 2}$ \\
& ECSSD & 5.1836 & 2.0232 & 3.8338 & 1.3300 & $\mathbf{9 . 0 4 6 0}$ \\
& ZuBuD & 3.2222 & 1.5889 & 2.7548 & 1.6068 & $\mathbf{9 . 0 2 5 4}$ \\
& 8-Scenes & 6.1928 & 2.1223 & 4.8818 & 1.3169 & $\mathbf{9 . 8 7 6 4}$ \\
\hline \multirow{4}{*}{$Q_{\text {sf }}$} & Ehinger & 30.9308 & 24.6578 & 27.7863 & 19.5718 & $\mathbf{5 2 . 2 8 7 1}$ \\
& ECSSD & 35.5826 & 32.6334 & 30.8033 & 17.1888 & $\mathbf{5 4 . 2 3 3 0}$ \\
& ZuBuD & 26.9374 & 28.8558 & 25.4766 & 18.9989 & $\mathbf{5 0 . 5 8 7 5}$ \\
& 8-Scenes & 38.9960 & 34.8072 & 34.8934 & 17.7691 & $\mathbf{5 9 . 4 0 7 7}$ \\
\hline \multirow{5}{*}{$Q_{g}$} & Ehinger & 14.0253 & 9.2482 & 12.7844 & 8.4133 & $\mathbf{2 1 . 5 6 3 9}$ \\
& ECSSD & 17.8250 & 12.9802 & 15.6790 & 8.0520 & $\mathbf{2 3 . 9 3 9 6}$ \\
& ZuBuD & 12.6257 & 10.9575 & 12.0646 & 8.5465 & $\mathbf{2 0 . 7 5 1 0}$ \\
& 8-Scenes & 18.1404 & 13.3453 & 16.4043 & 7.5928 & $\mathbf{2 4 . 6 5 9 8}$ \\
\hline \multirow{5}{*}{} & Ehinger & 0.3685 & 0.2914 & 0.3605 & 0.3325 & $\mathbf{0 . 4 8 1 7}$ \\
& ECSSD & 1.8378 & 1.3892 & 1.4356 & 0.8385 & $\mathbf{2 . 1 6 5 0}$ \\
& ZuBuD & 1.4127 & 1.3430 & 1.2603 & 0.9212 & $\mathbf{2 . 3 1 1 0}$ \\
& 8-Scenes & 1.9100 & 1.2879 & 1.4729 & 0.7931 & $\mathbf{2 . 2 3 0 9}$ \\
\hline \multirow{5}{*}{$Q_{\text {jnd }}$} & Ehinger & 1.5797 & 1.0689 & 1.2960 & 0.8881 & $\mathbf{2 . 2 5 0 0}$ \\
& ECSSD & 0.3267 & 0.3048 & 0.3123 & 0.2469 & $\mathbf{0 . 4 2 5 4}$ \\
& ZuBuD & 0.3276 & 0.3428 & 0.3240 & 0.2934 & $\mathbf{0 . 4 7 3 0}$ \\
& 8-Scenes & 0.3736 & 0.3244 & 0.3712 & 0.3082 & $\mathbf{0 . 4 8 1 6}$ \\
\hline
\end{tabular}

Table 4: Best Performances Rate of LSCN

\begin{tabular}{llccccc}
\hline & Number & $Q_{c}$ & $Q_{\text {sf }}$ & $Q_{g}$ & $\bar{r}$ & $Q_{\text {jnd }}$ \\
\hline Ehinger & 912 & 0.9934 & 0.9923 & 0.9879 & 0.9846 & 0.9485 \\
ECSSD & 1000 & 0.9070 & 0.9490 & 0.9090 & 0.8090 & 0.8930 \\
ZuBuD & 1005 & 0.9970 & 0.9950 & 0.9970 & 0.9662 & 0.9741 \\
8-Scenes & 2688 & 0.9286 & 0.9762 & 0.9442 & 0.7798 & 0.8865 \\
\hline
\end{tabular}


improved performance of the proposed method via subjective evaluation and quantitative assessment.

In the image enhancement algorithm, the details are enhanced to improve the visual appearance, but some noisy pixels are also magnified. What is more, because of using high-pass filter, there is very small ringing effect in LSCN results. In the future, we would like to improve the algorithm to solve these potential problems and to speed up the LSCN implementation. We will extend LSCN to other pixel-level image processing application such as image segmentation and medical image fusion.

\section{Acknowledgment}

This work has been supported by the National Science Foundation of China under the Grant No. 61201422, the Specialized Research Fund for the Doctoral Program of Higher Education under the Grant No. 20120211120013, and the Fundamental Research Funds for the Central Universities under the Grant No. lzujbky-2016-139 and lzujbky-2016-239.

\section{Reference}

[1] R. Eckhorn, R. Bauer, W. Jordan, M. Brosch, W. Kruse, M. Munk, H. Reitboeck, Coherent oscillations: A mechanism of feature linking in the visual cortex?, Biological Cybernetics 60 (2) (1988) 121-130.

[2] C. M. Gray, P. König, A. K. Engel, W. Singer, Oscillatory responses in cat visual cortex exhibit inter-columnar synchronization which reflects global stimulus properties, Nature 338 (6213) (1989) 334-337.

[3] P. Fries, Neuronal gamma-band synchronization as a fundamental process in cortical computation, Annual Review of Neuroscience 32 (2009) 209-224.

[4] G. Buzsáki, X.-J. Wang, Mechanisms of gamma oscillations, Annual Review of Neuroscience 35 (2012) 203-225.

[5] N. M. Brunet, C. A. Bosman, M. Vinck, M. Roberts, R. Oostenveld, R. Desimone, P. De Weerd, P. Fries, Stimulus repetition modulates gamma-band synchronization in primate visual cortex, Proceedings of the National Academy of Sciences 111 (9) (2014) 3626-3631. 
[6] R. Eckhorn, H. Reitboeck, M. Arndt, P. Dicke, Feature linking via synchronization among distributed assemblies: Simulations of results from cat visual cortex, Neural Computation 2 (3) (1990) 293-307.

[7] J. L. Johnson, D. Ritter, Observation of periodic waves in a pulsecoupled neural network, Optics letters 18 (15) (1993) 1253-1255.

[8] J. L. Johnson, J. R. Taylor, M. Anderson, Pulse-coupled neural network shadow compensation, Vol. 3722, 1999, pp. 452-456.

[9] J. L. Johnson, M. L. Padgett, PCNN models and applications., IEEE Transactions on Neural Networks 10 (3) (1999) 480-498.

[10] Y. Ma, K. Zhan, Z. Wang, Applications of Pulse-Coupled Neural Networks, Springer, Berlin, Germany, 2010.

[11] T. Lindblad, J. Kinser, Image Processing using Pulse-Coupled Neural Networks: Applications in Python, Springer, Berlin, Germany, 2013.

[12] L. Abbott, W. G. Regehr, Synaptic computation, Nature 431 (7010) (2004) 796-803.

[13] J. Li, H. Zhang, D. Yuan, M. Sun, Single image dehazing using the change of detail prior, Neurocomputing 156 (2015) 1 - 11.

[14] L. Xiao, C. Li, Z. Wu, T. Wang, An enhancement method for X-ray image via fuzzy noise removal and homomorphic filtering, Neurocomputing 195 (2016) $56-64$.

[15] B. Li, W. Xie, Image denoising and enhancement based on adaptive fractional calculus of small probability strategy, Neurocomputing 175-A (2016) $704-714$.

[16] Z. Chen, B. R. Abidi, D. L. Page, M. A. Abidi, Gray-level grouping (GLG): an automatic method for optimized image contrast enhancement-part I: the basic method, IEEE Transactions on Image Processing 15 (8) (2006) 2290-2302.

[17] T. Arici, S. Dikbas, Y. Altunbasak, A histogram modification framework and its application for image contrast enhancement, IEEE Transactions on Image Processing 18 (9) (2009) 1921-1935. 
[18] D. Sen, S. K. Pal, Automatic exact histogram specification for contrast enhancement and visual system based quantitative evaluation, IEEE Transactions on Image Processing 20 (5) (2011) 1211-1220.

[19] X. Wu, A linear programming approach for optimal contrast-tone mapping, IEEE Transactions on Image Processing 20 (5) (2011) 1262-1272.

[20] T. Celik, T. Tjahjadi, Automatic image equalization and contrast enhancement using gaussian mixture modeling, IEEE Transactions on Image Processing 21 (1) (2012) 145-156.

[21] H. Xu, G. Zhai, X. Wu, X. Yang, Generalized equalization model for image enhancement, IEEE Transactions on Multimedia 16 (1) (2014) 68-82.

[22] J.-L. Starck, F. Murtagh, E. J. Candes, D. L. Donoho, Gray and color image contrast enhancement by the curvelet transform, IEEE Transactions on Image Processing 12 (6) (2003) 706-717.

[23] J. Tang, J. Kim, E. Peli, Image enhancement in the JPEG domain for people with vision impairment, IEEE Transactions on Biomedical Engineering 51 (11) (2004) 2013-2023.

[24] J. Mukherjee, S. K. Mitra, Enhancement of color images by scaling the dct coefficients, IEEE Transactions on Image Processing 17 (10) (2008) 1783-1794.

[25] P. Perona, J. Malik, Scale-space and edge detection using anisotropic diffusion, IEEE Transactions on Pattern Analysis and Machine Intelligence 12 (7) (1990) 629-639.

[26] C. Tomasi, R. Manduchi, Bilateral filtering for gray and color images, in: Proc. of ICCV, Vol. 6, IEEE, 1998, pp. 839-846.

[27] Z. Farbman, R. Fattal, D. Lischinski, R. Szeliski, Edge-preserving decompositions for multi-scale tone and detail manipulation, in: ACM Transactions on Graphics (TOG), Vol. 27, ACM, 2008, p. 67.

[28] L. Xu, C. Lu, Y. Xu, J. Jia, Image smoothing via 10 gradient minimization, in: ACM Transactions on Graphics (TOG), Vol. 30, ACM, 2011, p. 174 . 
[29] K. He, J. Sun, X. Tang, Guided image filtering, IEEE Transactions on Pattern Analysis and Machine Intelligence 35 (6) (2013) 1397-1409.

[30] H. Xu, G. Zhai, X. Yang, Single image super-resolution with detail enhancement based on local fractal analysis of gradient, IEEE Transactions on Circuits and Systems for Video Technology 23 (10) (2013) 1740-1754.

[31] F. Zhang, L. Dai, S. Xiang, X. Zhang, Segment graph based image filtering: Fast structure-preserving smoothing, in: Proc. of ICCV, IEEE, 2015, pp. 361-369.

[32] E. H. Land, The retinex theory of color vision, Scientific American 237 (6) (1977) 108-128.

[33] S. C. Nercessian, K. Panetta, S. S. Agaian, Non-linear direct multiscale image enhancement based on the luminance and contrast masking characteristics of the human visual system, IEEE Transactions on Image Processing 22 (9) (2013) 3549-3561.

[34] Y. Wang, H. Wang, C. Yin, M. Dai, Biologically inspired image enhancement based on retinex, Neurocomputing 177 (2016) 373-384.

[35] K. Zhan, H. Zhang, Y. Ma, New spiking cortical model for invariant texture retrieval and image processing, IEEE Transactions on Neural Networks 20 (12) (2009) 1980-1986.

[36] Y. Ma, F. Teng, K. Zhan, H. Zhang, A new method of color image enhancement using spiking cortical model, Journal of Beijing University of Posts and Telecommunications 35 (3) (2012) 70-73.

[37] R. C. Gonzalez, R. E. Woods, Digital Image Processing (3rd Edition), Pearson Education, London, UK, 2007.

[38] Y. Gong, I. F. Sbalzarini, Image enhancement by gradient distribution specification, in: Computer Vision-ACCV 2014 Workshops, Springer, 2014, pp. 47-62.

[39] T. Brosch, H. Neumann, Interaction of feedforward and feedback stream$\mathrm{s}$ in visual cortex in a firing-rate model of columnar computations, Neural Networks 54 (2014) 11-16. 
[40] A. S. French, R. B. Stein, A flexible neural analog using integrated circuits, IEEE Transactions on Biomedical Engineering BME-17 (3) (1970) 248-253.

[41] J.-S. Lee, Digital image enhancement and noise filtering by use of local statistics, IEEE Transactions on Pattern Analysis and Machine Intelligence (2) (1980) 165-168.

[42] D. T. Kuan, A. A. Sawchuk, T. C. Strand, P. Chavel, Adaptive noise smoothing filter for images with signal-dependent noise, IEEE Transactions on Pattern Analysis and Machine Intelligence (2) (1985) 165-177.

[43] K. B. Gibson, T. Q. Nguyen, Fast single image fog removal using the adaptive wiener filter, in: Proc. of ICIP, Vol. 20, IEEE, 2013, pp. 714718.

[44] C. C. Pham, S. V. U. Ha, J. W. Jeon, Adaptive guided image filtering for sharpness enhancement and noise reduction, in: Advances in Image and Video Technology, Springer, 2011, pp. 323-334.

[45] Z. Li, J. Zheng, Z. Zhu, W. Yao, S. Wu, Weighted guided image filtering, IEEE Transactions on Image Processing 24 (1) (2015) 120-129.

[46] H. S. Ranganath, G. Kuntimad, J. L. Johnson, Pulse coupled neural networks for image processing, in: Proc. of Southeastcon'95 Visualize the Future, IEEE, 1995, pp. 37-43.

[47] F. De Vries, Automatic, adaptive, brightness independent contrast enhancement, Signal Processing 21 (2) (1990) 169-182.

[48] A. M. Eskicioglu, P. S. Fisher, Image quality measures and their performance, IEEE Transactions on Communications 43 (12) (1995) 29592965 .

[49] X. Bai, Y. Zhang, Enhancement of microscopy mineral images through constructing alternating operators using opening and closing based toggle operator, Journal of Optics 16 (12) (2014) 125407.

[50] N. Hautiere, J.-P. Tarel, D. Aubert, E. Dumont, et al., Blind contrast enhancement assessment by gradient ratioing at visible edges, Image Analysis \& Stereology Journal 27 (2) (2008) 87-95. 
[51] X. Wang, M. Zeng, A new metric for objectively assessing the quality of enhanced images based on human visual perception, Journal of Optoelectronics Laser 19 (2) (2008) 258-262.

[52] E. Peli, Contrast in complex images, Journal Optical Society of America A 7 (10) (1990) 2032-2040.

[53] C.-H. Chou, Y.-C. Li, A perceptually tuned subband image coder based on the measure of just-noticeable-distortion profile, IEEE Transactions on Circuits and Systems for Video Technology 5 (6) (1995) 467-476.

[54] X. Yang, W. Ling, Z. Lu, E. P. Ong, S. Yao, Just noticeable distortion model and its applications in video coding, Signal Processing: Image Communication 20 (7) (2005) 662-680.

[55] P. Arbelaez, M. Maire, C. Fowlkes, J. Malik, Contour detection and hierarchical image segmentation, IEEE Transactions on Pattern Analysis and Machine Intelligence 33 (5) (2011) 898-916.

[56] K. A. Ehinger, B. Hidalgo-Sotelo, A. Torralba, A. Oliva, Modelling search for people in 900 scenes: A combined source model of eye guidance, Visual Cognition 17 (6-7) (2009) 945-978.

[57] Q. Yan, L. Xu, J. Shi, J. Jia, Hierarchical saliency detection, in: Proc. of CVPR, IEEE, 2013, pp. 1155-1162.

[58] A. Oliva, A. Torralba, Modeling the shape of the scene: A holistic representation of the spatial envelope, International Journal of Computer Vision 42 (3) (2001) 145-175.

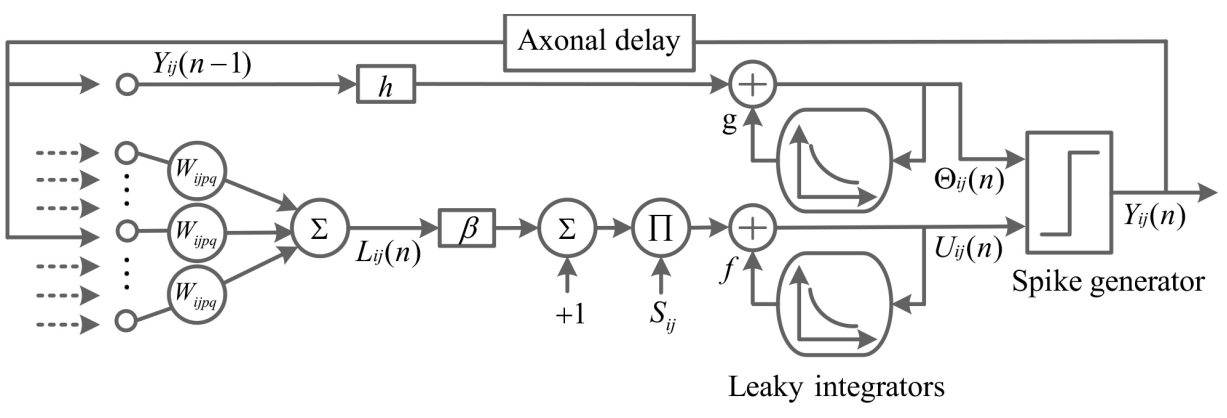

Figure 1: Schematic of Linking Synaptic Computation Network. 


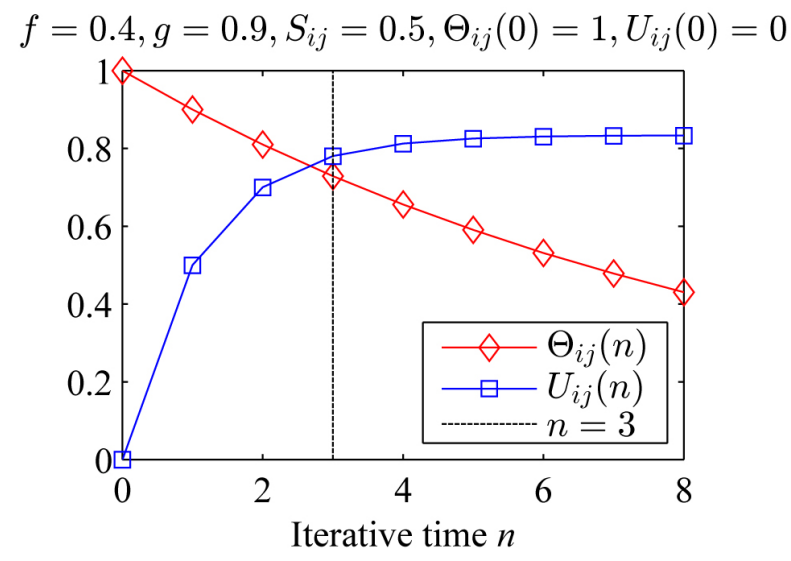

Figure 2: The first spiking time of a neuron in a specific example

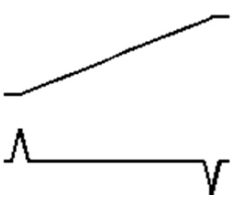

Figure 3: The first line is an edge of the stimulus signal, and the second line shows that the first line is filtered by the Laplacian operator. 


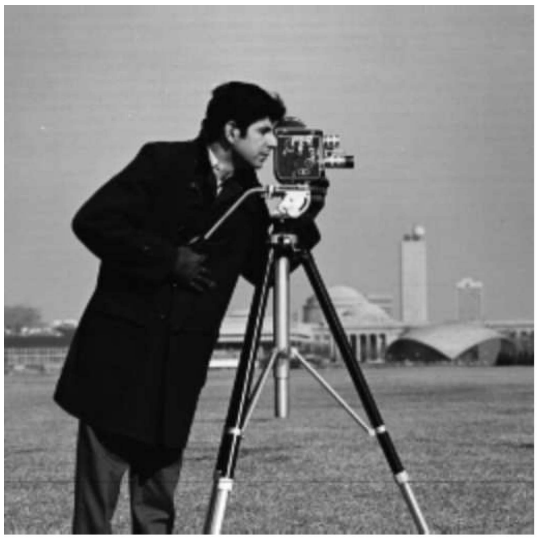

(a)

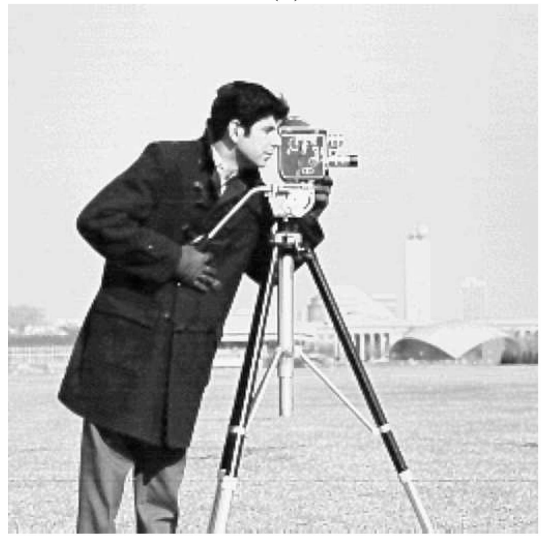

(c)

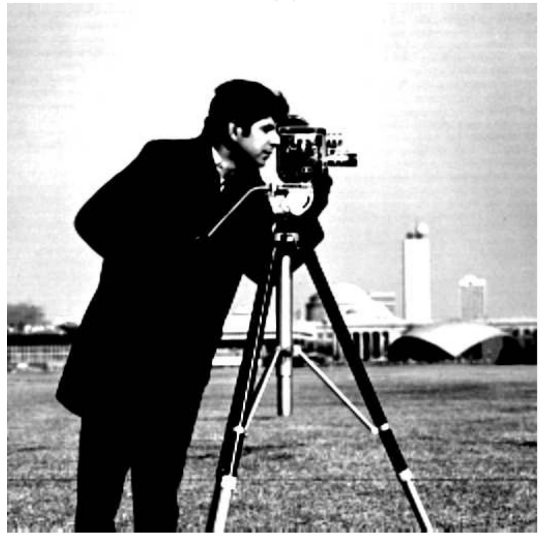

(e)

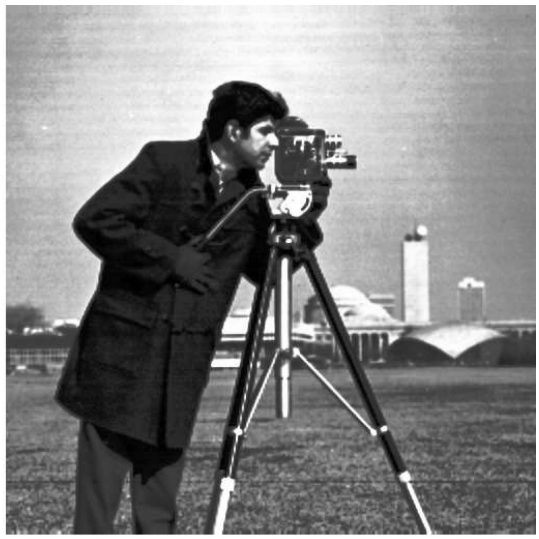

(b)

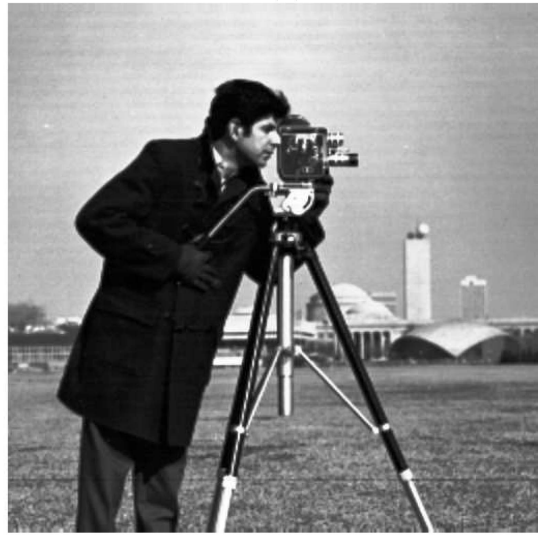

(d)

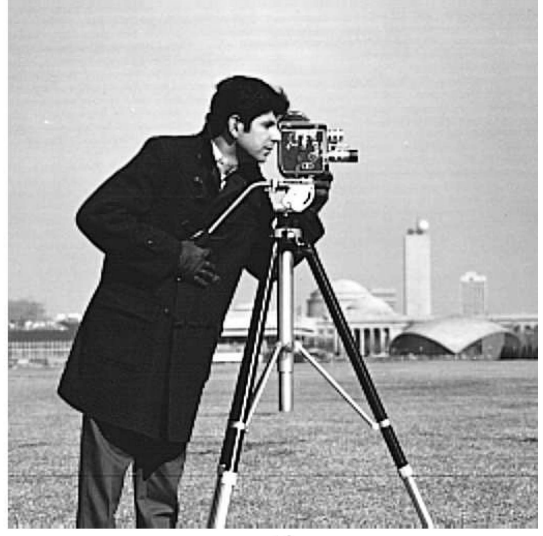

(f)

Figure 4: Input image and enhanced images obtained by different methods, (a) man, (b) HEQ, (c) SCM, (d) GEM, (e) GDS, (f) LSCN. 


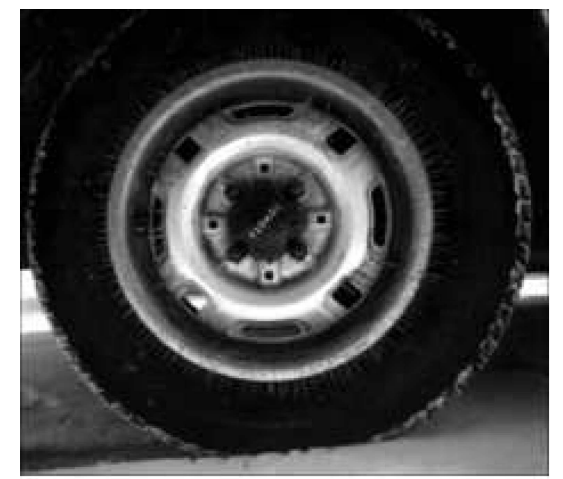

(a)

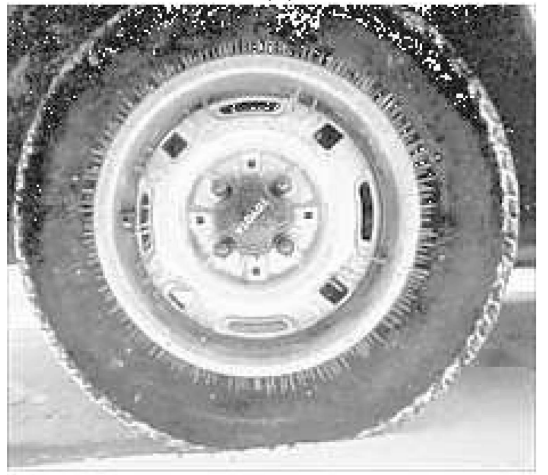

(c)

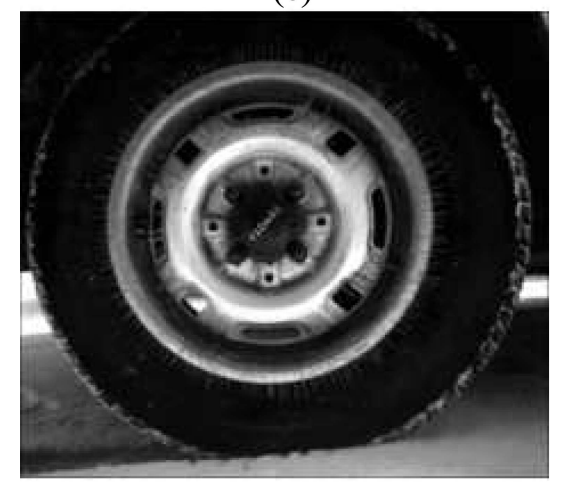

(e)

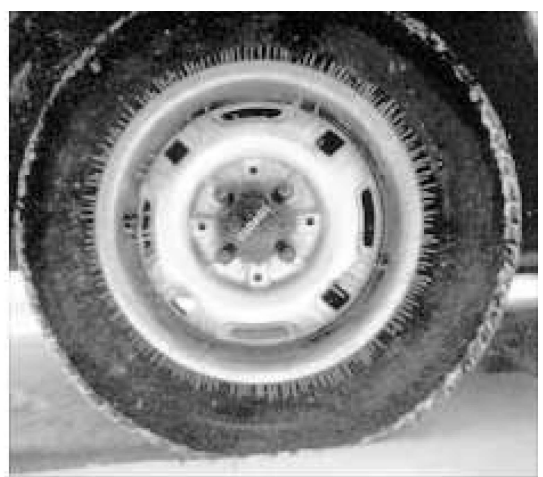

(b)

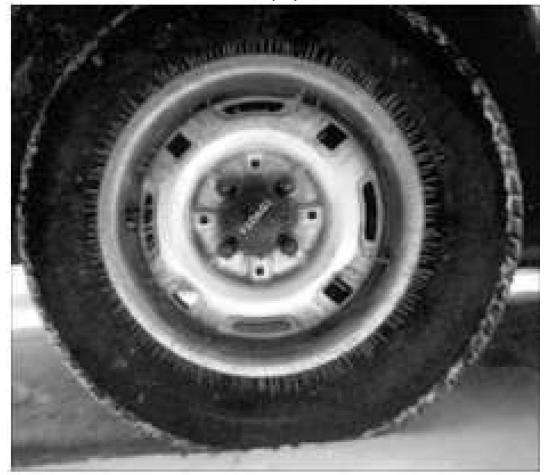

(d)

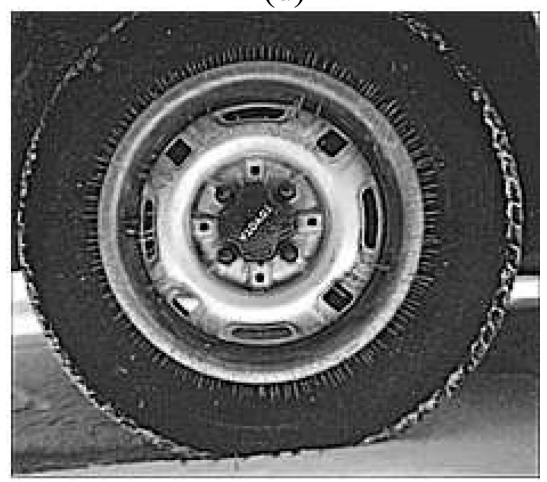

(f)

Figure 5: Input image and enhanced images obtained by different methods, (a) tire, (b) HEQ, (c) SCM, (d) GEM, (e) GDS, (f) LSCN. 


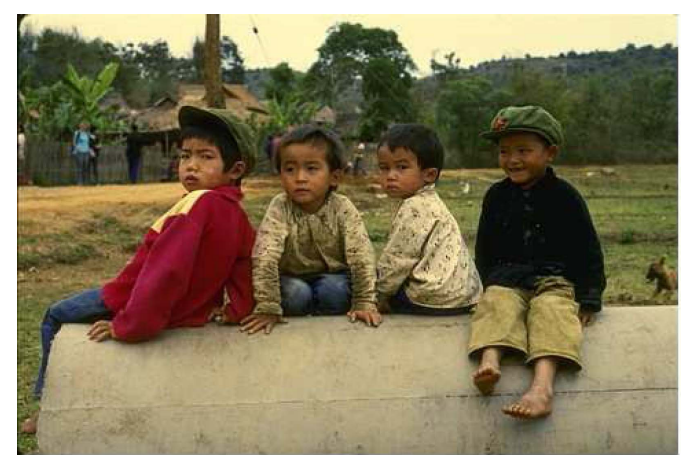

(a)

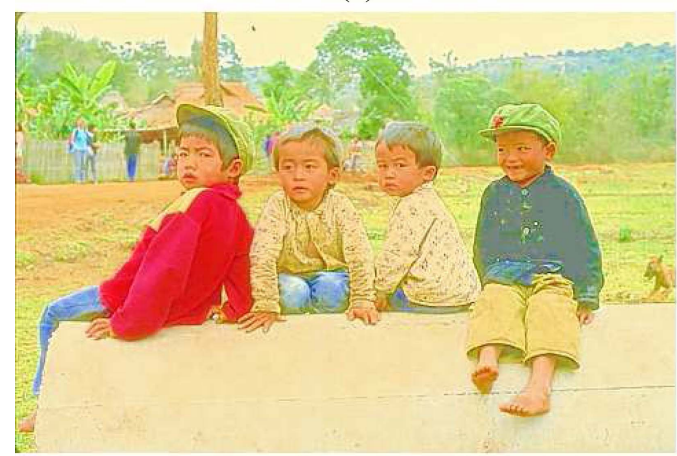

(c)

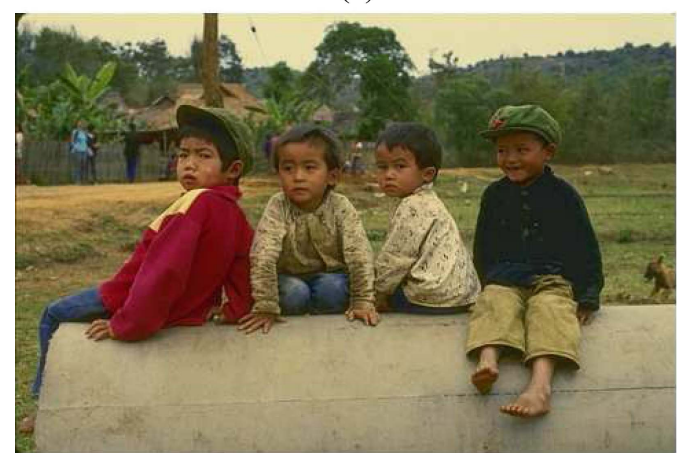

(e)

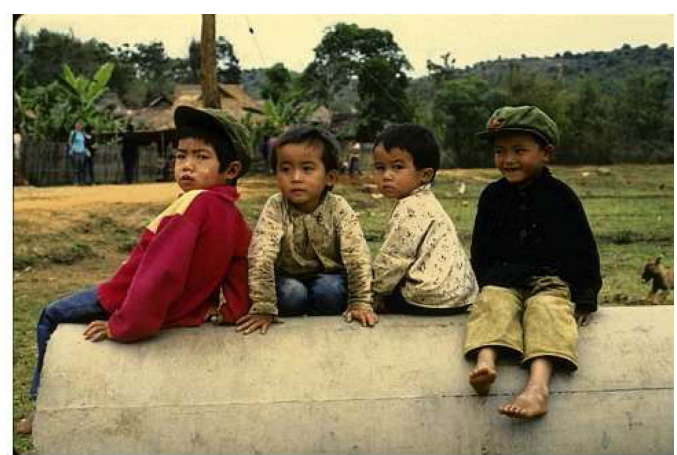

(b)

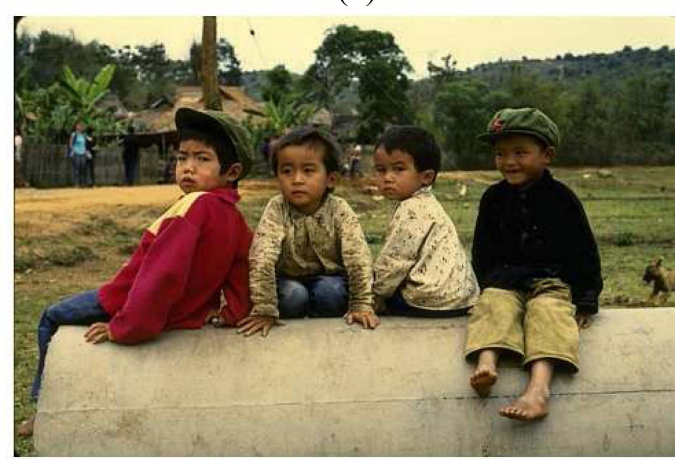

(d)

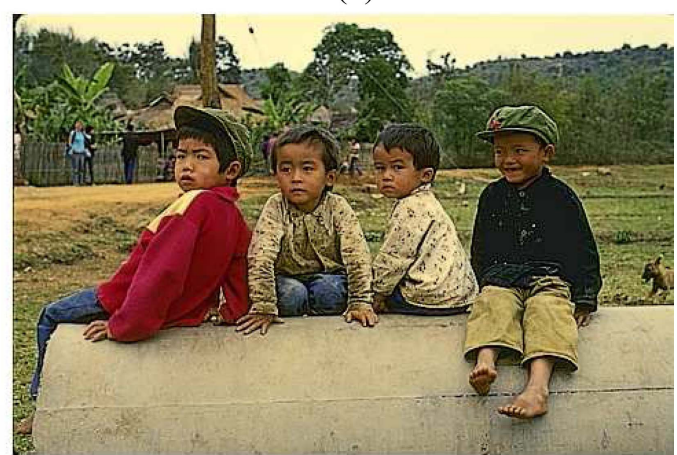

(f)

Figure 6: Input image and enhanced images obtained by different methods, (a) boys, (b) HEQ, (c) SCM, (d) GEM, (e) GDS, (f) LSCN. 


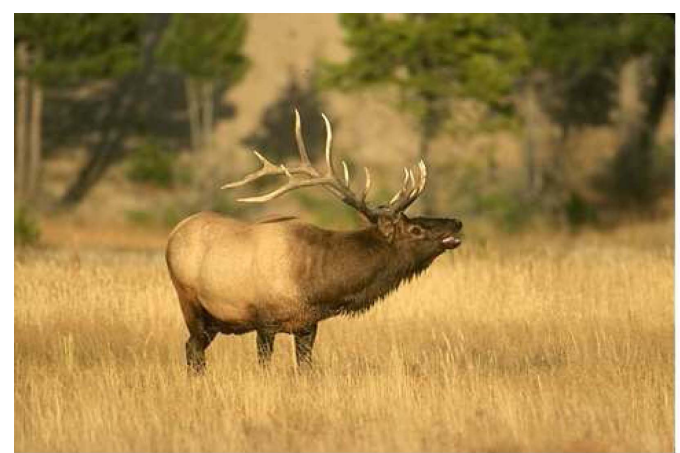

(a)

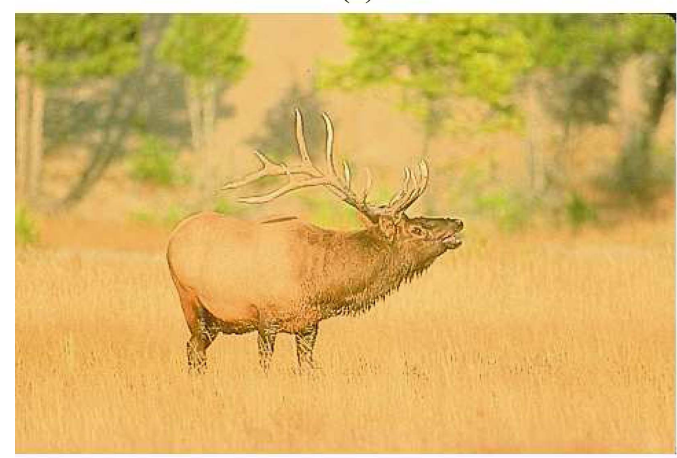

(c)

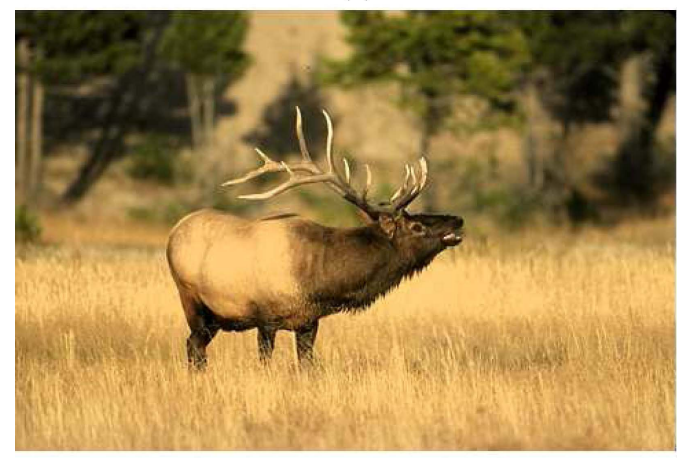

(e)

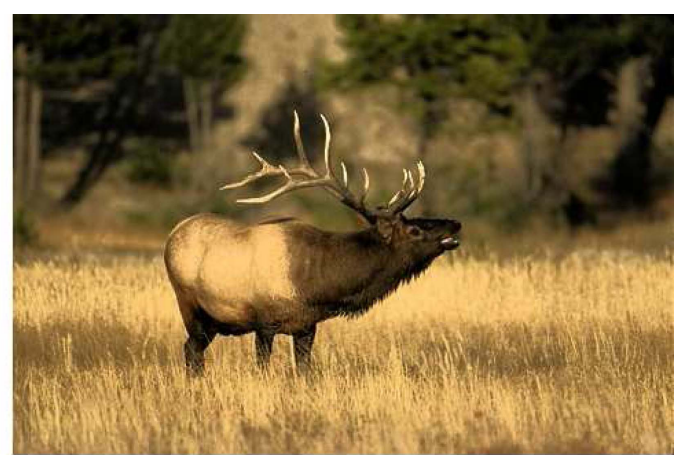

(b)

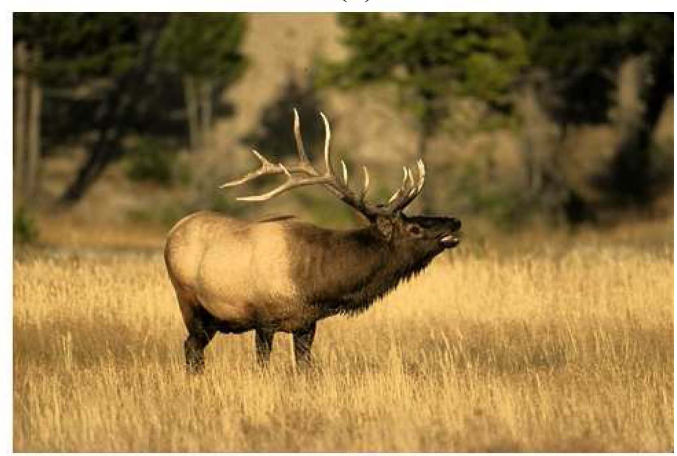

(d)

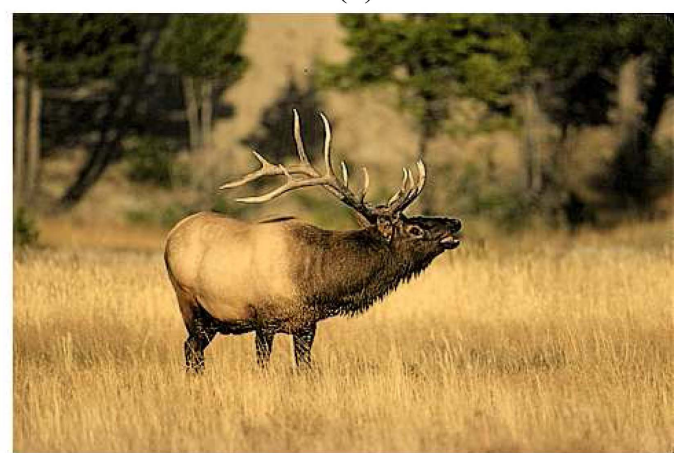

(f)

Figure 7: Input image and enhanced images obtained by different methods, (a) deer, (b) HEQ, (c) SCM, (d) GEM, (e) GDS, (f) LSCN. 


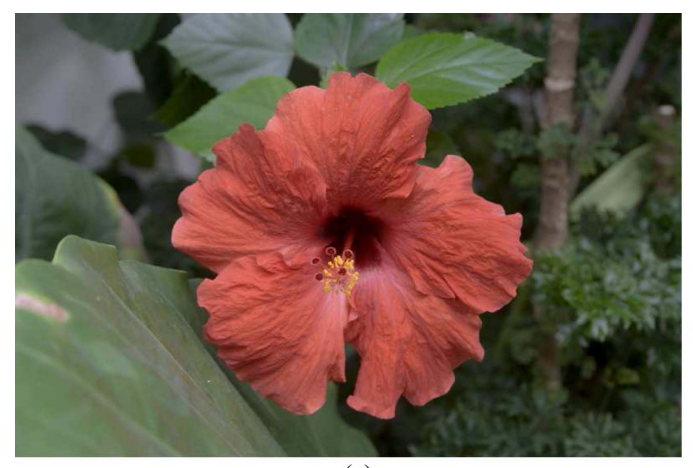

(a)
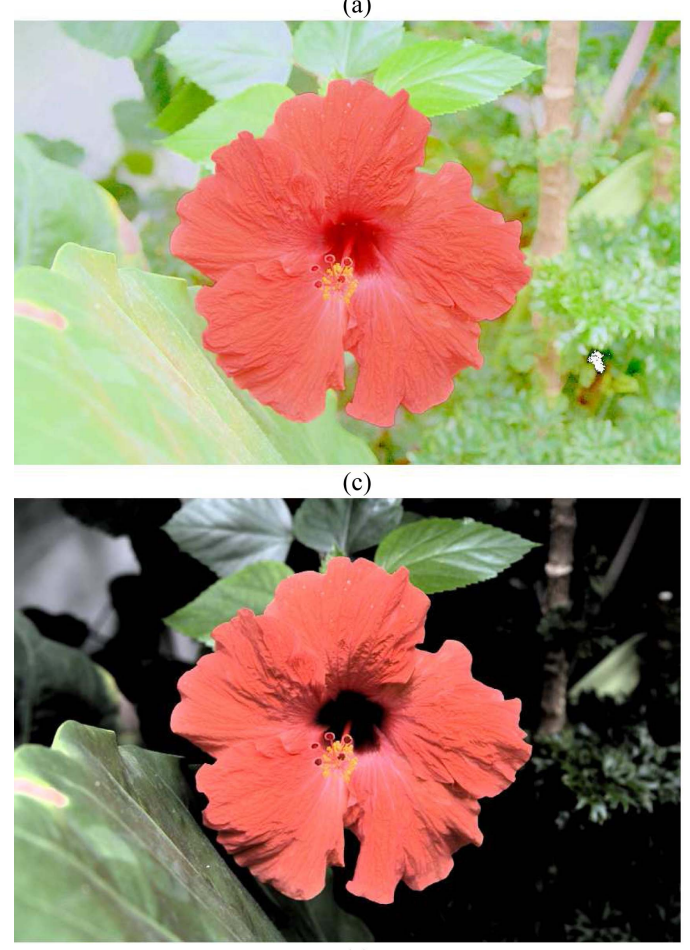

(e)

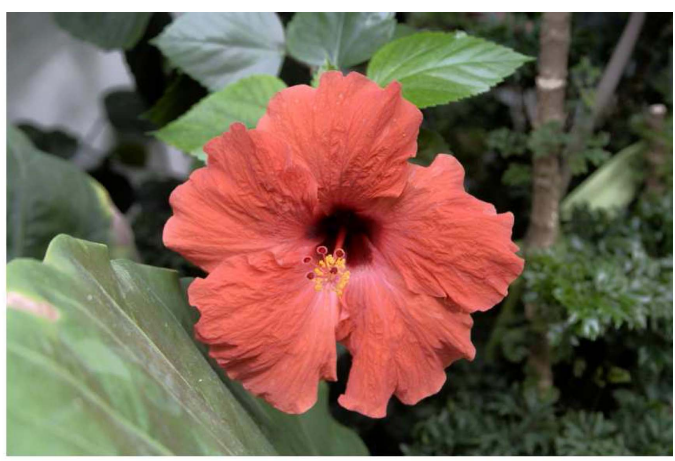

(b)

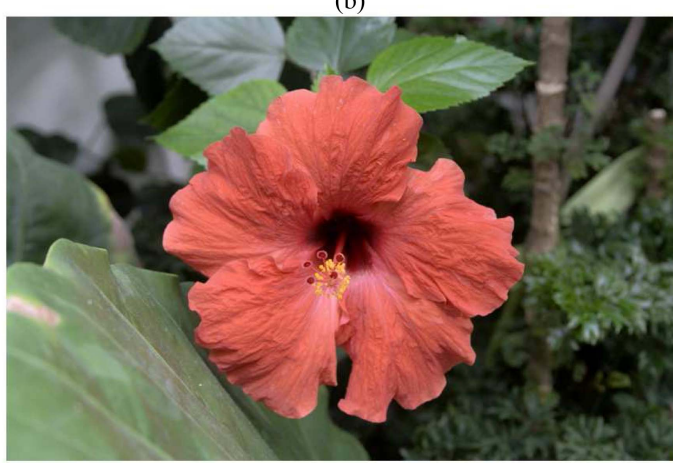

(d)

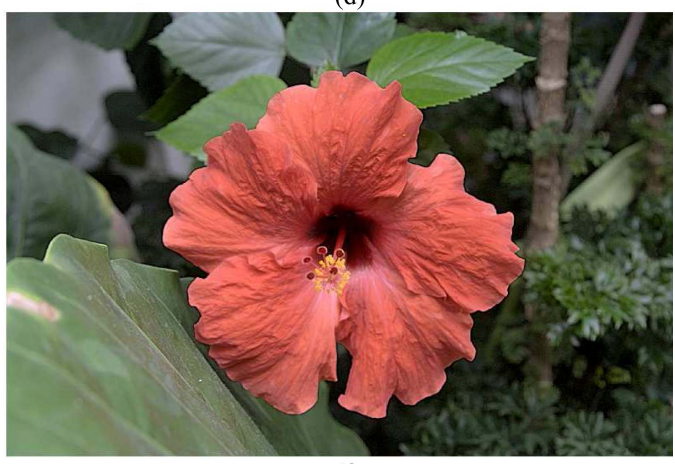

(f)

Figure 8: Input image and enhanced images obtained by different methods, (a) flower, (b) HEQ, (c) SCM, (d) GEM, (e) GDS, (f) LSCN. 

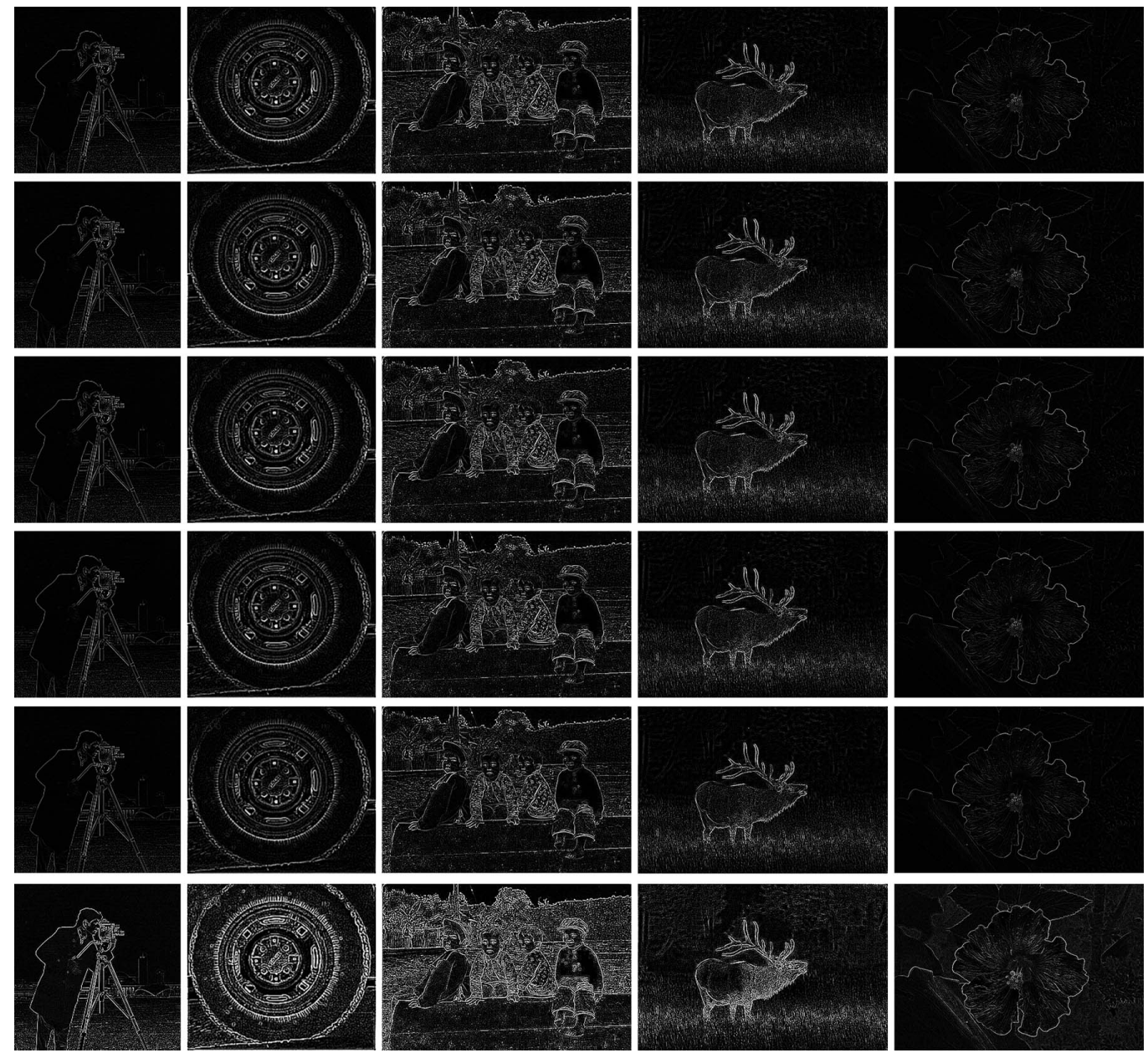

Figure 9: Edge Detection Results. From top to bottom: inputs, HEQ outputs, SCM outputs, GEM outputs, GDS outputs, and LSCN outputs, respectively. 